www.scientiaplena.org.br

doi: 10.14808/sci.plena.2017.061202

\title{
Saberes relacionados ao uso de plantas medicinais e influência na prática didática dos estudantes de Mãe D’Água, Paraíba, Brasil
}

\author{
Knowledge related to the use of medicinal plants and influence in the didactic practice of students \\ from Mãe D'Água, Paraíba, Brazil \\ M. A. F. S. Lustosa*; L. A. Santos; R. M. de Araújo; G. C. da Silva \\ M. G. V. Marinho; E. Silva \\ Unidade Acadêmica de Ciências Biológicas/Centro de Saúde e Tecnologia Rural, Universidade Federal de Campina \\ Grande, CEP 58708-110, Patos-PB, Brasil \\ *cidafelix15@hotmail.com
}

(Recebido em 26 de janeiro de 2017; aceito em 29 de junho de 2017)

\begin{abstract}
Esta pesquisa objetivou avaliar os conhecimentos e envolvimento dos estudantes do ensino médio público (1520 anos) da cidade de Mãe D'Água, Paraíba, relacionados às plantas medicinais e promover a construção de saberes nessa área por meio de uma prática de ensino. O estudo teve três momentos distintos: aplicação de questionário constituído 9 afirmativas estruturadas segundo o modelo da escala de Likert e mais 6 perguntas; vivência prática didática com os alunos e; reaplicação do questionário. Os alunos possuíam pouco conhecimento sobre A etnobotânica, plantas medicinais e o seu uso. Entretanto, após a vivência didática, se observou aumento significativo desse conhecimento, tais como conceituar corretamente a Etnobotânica e o que são plantas medicinais. Os conhecimentos sobre plantas medicinais foram adquiridos, principalmente, dos avós. As folhas é a parte que eles mais usam para fins medicinais. Antes da prática, eles $24,5 \%$ acreditavam que as plantas medicinais não causavam danos à saúde, $67,3 \%$ consideravam os seus efeitos moderados. Após a prática didática, esses percentuais modificaram-se para $8,2 \%$ e $51 \%$, respectivamente. A prática didática foi eficaz para mediar e melhorar esse conhecimento. Os alunos, antes e após a prática didática, consideraram importante o estudo das plantas medicinais na disciplina de Biologia para informar sobre seus diferentes aspectos, tais como: ecológico, botânico (morfológico e taxonômico). É veemente a necessidade da inserção do ensino sobre plantas medicinais no currículo escolar, para que haja a construção do saber e promoção da valorização e uso dessas plantas presentes na comunidade em que os estudantes vivem.

Palavras chaves: Atividades didáticas, Conhecimentos, Etnobotânica
\end{abstract}

This research aimed to evaluate the knowledge and involvement of the public high school students (15-20 years) from the city of Mãe D'Água, Paraíba, related to medicinal plants and to promote the construction of knowledge in this area through a teaching practice. The study had three distinct moments: questionnaire application consisting of 9 affirmations structured according to the Likert scale model and 6 questions; Practical didactic experience with the students and; Reapplication of the questionnaire. The students had little knowledge about ethnobotany, medicinal plants and their use. However, after the didactic experience, a significant increase of this knowledge was observed, such as correctly conceptualizing Ethnobotany and what are medicinal plants. Knowledge about medicinal plants was mainly acquired from grandparents. The leaves are the part they most use for medicinal purposes. Before the practice, $24.5 \%$ believed that medicinal plants did not cause health damage, $67.3 \%$ considered their effects to be moderate. After the didactic practice, these percentages changed to $8.2 \%$ and $51 \%$, respectively. Didactic practice was effective in mediating and improving this knowledge. The students, before and after didactic practice, considered important the study of medicinal plants in the discipline of Biology to inform about its different aspects, such as: ecological, botanical (morphological and taxonomic). There is a strong need for the inclusion of teaching on medicinal plants in the school curriculum, so that there is the construction of knowledge and promotion of the valuation and use of these plants present in the community in which the students live.

Keywords: Didactic activities, Knowledge, Ethnobotany 


\section{INTRODUÇÃO}

A Etnobotânica estuda a interação existente entre o ser humano e as plantas bem como as formas de sua utilização [1], apresentando caráter interdisciplinar no ambiente educacional, com a promoção ações e trocas de conhecimento empírico entre a ciência e a sociedade [2]. Os estudos Etnobotânicos também se destacam como sendo relevantes para popularização da ciência, valorização da economia, benefícios à sociedade e para conservação da vegetação em diversas regiões brasileiras [3]. O estudo das plantas medicinais é importante para registro de informação, na identificação de espécies e no avanço econômico e de uso medicinal das plantas [4]. Seu uso acompanha o ser humano desde a antiguidade [5] e a problemática da falta de acesso à saúde pública, possibilita o uso de plantas medicinais pela comunidade [6].

A mudança cultural, o desenvolvimento das cidades e a alteração na forma de comunicação entre as pessoas, podem causar perca do conhecimento que existem sobre às plantas medicinais [7]. De acordo com as diretrizes da Política Nacional de plantas medicinais e Fitoterápicos, implantadas em 2006, a educação sobre plantas medicinais deve ser inserida em todos os níveis de ensino formal [8]. Essa normativa tem o intuito de garantir o acesso seguro e o uso racional de plantas medicinal e fitoterápico no Brasil. E, nesse contexto educacional, as escolas possuem papel fundamental para mediar esse conhecimento aos alunos [9].

A escola é o ambiente articulador do conhecimento em diversas áreas, apresenta o propósito sociocultural e dialético com interesse de desenvolver no aluno interpretações de mundo, de cultura e assim formar indivíduos críticos da sua realidade [10]. Para minimizar a problemática da perda desse conhecimento, a escola é um ambiente social importante na disseminação do conhecimento, pois, o trabalho sobre plantas medicinais favorece o processo de ensino e aprendizagem envolvendo o educando na instrução do saber.

Os docentes também apresentam papel fundamental no desenvolvimento das práticas didáticas que estabeleçam relações entre o aluno e o meio onde ele está inserido, podendo assim, sistematizar a distância entre esses saberes e favorecer a relação entre o ensino/aprendizagem e proporcionar ao aluno a construção do conhecimento [11]. A prática de ensino relacionado a plantas são considerados sem relevância para os estudantes devido à utilização das práticas pedagógicas tradicionais [12]. Diante desta problemática outros métodos de ensino tais como a prática de ensino com diálogos e exposição é utilizada para melhorar significativamente o conhecimento do aluno.

Desse modo, é relevante a execução de pesquisas voltadas para práticas de ensino inovadoras para auxiliar os docentes na abordagem de temas transversais e/ou interdisciplinar e assim torná-lo mais abrangente e fortalecedor no plano da percepção em saberes Etnobotânicos. Neste contexto esta pesquisa teve como objetivo avaliar os conhecimentos e envolvimento dos estudantes do ensino médio público da cidade de Mãe D’Água, Paraíba, relacionados às plantas medicinais e promover a construção de saberes nessa área por meio de uma prática de ensino.

\section{MATERIAL E MÉTODOS}

\section{1 Área de estudo, População e Amostra}

O município de Mãe D’Água está localizado (07² 15 '27.81’S e $037^{\circ} 25^{\prime} 36.66^{\prime}$ ' O) no bioma Caatinga, na mesorregião do Sertão do Paraibano e apresenta uma área de $246 \mathrm{Km}^{2}$ e sua população é de aproximadamente 4 mil habitantes IBGE [13].

A pesquisa foi realizada de 23 a 30 de março de 2016 na Escola Estadual de Ensino Fundamental e Médio Francisco Romano da Silveira, situada na região oeste do município de Mãe D’Água, Paraíba. A população amostral foi definida, a partir do total de estudantes matriculados na escola (N =152) e considerando um erro padrão igual a 10\% [14]. Dessa forma, a amostragem constituiu de 49 alunos. Os critérios de inclusão para a seleção dos alunos foram: estar cursando o $2^{\circ}$ e $3^{\circ}$ ano do ensino médio da Escola Francisco Romano da Silveira, distribuídas em quatro turmas e ter aptidão 
física e cognitiva para ler e interpretar o questionário. Dentro desse critério, a amostragem dos alunos foi probabilística, aonde os alunos foram selecionados obedecendo à condição de aleatoriedade, em quatro salas de aulas.

\subsection{Procedimento metodológico}

Essa é uma pesquisa de natureza descritiva de caráter quali e quantitativo. Ela foi desenvolvida em três etapas distintas:

- Etapa 1: Aplicação de questionário: foi aplicado um questionário constituído por 6 perguntas (três discursivas e três objetivas) e nove itens segundo o modelo da escala de Likert, com 5 níveis de respostas que variam entre "discordo completamente (nível 1) a concordo completamente (nível 5)".

- Etapa 2: Vivência de prática didática: Temas relacionados à Etnobotânica e específicos sobre plantas medicinais, Plantas Tóxicas, Fitoterapia, efeitos colaterais das plantas medicinais e a importância da disciplina Biologia abordar temas relacionados às plantas medicinais nas áreas de Ecologia e Botânica para o estudo da Morfologia e Taxonomia. A prática foi uma exposição dialógica e expositiva desenvolvida segundo plano de ensino específico e com duração de 2 horas. Para melhor aplicação dessa prática didática, os alunos entrevistados foram divididos em 2 grupos e a atividade foi aplicada para ambos de maneira semelhante.

- Etapa 3: Reaplicação do questionário: Após uma semana da realização da prática de ensino houve a reaplicação do questionário aplicado na etapa 1 , com a finalidade de avaliar o nível de conhecimento adquirido pelos estudantes.

A prática educativa iniciou-se com um primeiro contato (dois dias antes do encontro para a vivência) com os estudantes para explicar o que seria desenvolvido para contribuir com o seu aprendizado e dessa maneira, envolvê-los e motivá-los a participarem.

A escala de Likert é um método que oferece direcionamento sobre a atitude do respondente em relação a cada afirmação, sendo está positiva ou negativa [15].

Essa pesquisa foi desenvolvida dentro de aspectos legais mediante Termo de Autorização assinado pelo responsável da instituição supracitada. Os alunos participantes foram esclarecidos quanto aos objetivos da pesquisa e a sua participação foi voluntária e anuída pelo termo de consentimento livre e esclarecido (TCLE).

\subsection{Análise dos dados}

Os dados do questionário foram analisados de maneira quali e qualitativamente por meio da estatística descritiva, utilizando Microsoft Excel 365. Para avaliar as respostas das perguntas qualitativas $O$ que é Etnobotânica? e $O$ que é uma planta medicinal?, foram utilizados os conceitos de acordo com [16, 17] para Etnobotânica e; para plantas medicinais, de acordo com a legislação vigente no Brasil (Tabela 1). De acordo com essa avaliação, as respostas foram classificadas e quantificadas, em percentual, como certa, certa em parte ou errada. 
Tabela 1: Definição conceitual para Etnobotânica e plantas medicinais utilizado para análise das respostas dos alunos entrevistados

\begin{tabular}{clll}
\hline Conceito & \multicolumn{1}{c}{ Definição } & Referência \\
\hline \multirow{2}{*}{ Etnobotânica } & $\begin{array}{l}\text { A Etnobotânica estuda a interação existente entre animais e planta bem } \\
\text { como a forma de sua utilização pelo homem, se importa com a } \\
\text { manutenção desse conhecimento. }\end{array}$ & \\
\hline \multirow{2}{*}{$\begin{array}{l}\text { Plantas } \\
\text { medicinais }\end{array}$} & $\begin{array}{l}\text { São plantas que contêm em suas partes ou em toda a planta compostos } \\
\text { fitoterápicos após passar pelos processos que as tornem próprias para } \\
\text { o uso. }\end{array}$ & \\
\hline
\end{tabular}

\section{RESULTADOS E DISCUSSÃO}

Dentre os entrevistados, $42,9 \%$ foram do gênero masculino e $57,1 \%$ do gênero feminino, com faixa etária entre 15 e 20 anos, onde $61,2 \%$ residem na zona urbana e 38,8\% na rural (Tabela 2).

Tabela 2: Frequências absolutas ( $f a$ ) e relativas ( $f r$ ) da região de moradia e faixa etária dos estudantes entrevistados

\begin{tabular}{lc|c}
\hline \multicolumn{1}{c}{ Variáveis } & fa & fr \\
\hline Região da Residência & & \\
Zona Rural & 19 & 38,8 \\
Zona Urbana & 30 & 61,2 \\
Total & 49 & 100 \\
\hline Faixa Etária (anos) & & \\
$15-16$ & 24 & 49,0 \\
$17-18$ & 22 & 44,9 \\
$>18$ & 3 & 6,1 \\
Total & 49 & 100 \\
\hline
\end{tabular}

Os estudantes $20,4 \%(n=10)$ afirmaram ter bom ou muito conhecimento sobre plantas medicinais e 4,1\% (n=2) demonstraram ter bom ou muito conhecimento sobre a temática de Plantas Tóxicas. Após a prática de ensino, esse percentual modificou-se, elevando-se para 71,4\% $(n=35)$ e 65,3\% (n $=32$ ), respectivamente (Tabela 3 ).

Dados similares foram observados por Cruz et al. (2009) [18] em sua pesquisa com alunos do ensino fundamental de cinco escolas da rede particular do município de São José dos Campos, onde o conhecimento prévio dos estudantes em relação as plantas medicinais eram limitado. Para os autores, esse fato é devido à pouca abordagem do tema em sala de aula. Dávila et al. (2016) [9] também reportaram resultados similares em pesquisa realizada com alunos de duas escolas públicas no município de Uruguaiana, Rio Grande do Sul. Foi verificado que a maior parte dos estudantes ainda não havia estudado no ambiente escolar temáticas relacionadas às plantas medicinais e/ou plantas tóxicas pois, dos 44 estudantes que participaram da pesquisa, 34\% $(\mathrm{n}=15)$ não conheciam nenhuma espécie de planta medicinal e 59,0\% $(\mathrm{n}=26)$ afirmaram não apresentar conhecimentos referente as plantas tóxicas.

Para que haja uma melhoria no ensino/aprendizagem dos estudantes sobre as plantas medicinais e tóxicas, é necessário a abordagem de temáticas na sala de aula, visto que essas plantas fazem parte do cotidiano dos estudantes, esses temas poderiam ser abordados em assuntos de biologia como: morfologia e anatomia vegetal na área de botânica, pois, para Veiga Junior et al. (2005) [19] é relevante alertar que plantas tóxicas que fazem parte do cotidiano popular, tais como o Juazeiro e o Avelós, provocam irritações que podem ocasionar complicações à saúde. O Sistema Nacional de 
Informações Tóxico Farmacológicas (SINTOX) registrou que em 2012 ocorreram 1.185 casos de intoxicação por plantas, sendo que 960 dos casos ocorreram em zonas urbanas [20].

Tabela 3: Frequência percentual de conhecimento dos alunos Escola Francisco Romano da Silva, antes e após, a prática didática desta pesquisa.

\begin{tabular}{lcc|c|c|c|c|c}
\hline \multirow{2}{*}{\multicolumn{1}{c}{ Item }} & \multicolumn{3}{c|}{ Antes } & \multicolumn{3}{c}{ Após } \\
\cline { 2 - 7 } & NPC & RC & BMC & NPC & RC & BMC \\
\hline Em relação às plantas medicinais & 38,8 & 40,8 & 20,4 & 8,2 & 20,4 & 71,4 \\
Em relação às plantas tóxicas & 81,6 & 14,3 & 4,1 & 6,1 & 28,6 & 65,3 \\
Sobre Etnobotânica & 89,8 & 10,2 & 0,0 & 16,3 & 38,8 & 51,0 \\
Cuidados no consumo de plantas medicinais & 49,0 & 26,5 & 24,5 & 8,2 & 8,2 & 81,6 \\
Em relação à Fitoterapia & 79,6 & 16,3 & 4,1 & 4,1 & 24,5 & 69,4 \\
\hline
\end{tabular}

Importância da Biologia para o ensino sobre plantas medicinais nos seguintes aspectos:

\begin{tabular}{lccc|ccr}
\hline Ecológico & 4,1 & 4,1 & 89,8 & 4,1 & 2,0 & 93,9 \\
Botânico & 4,1 & 6,1 & 89,8 & 4,1 & 2,0 & 93,9 \\
Taxonômico & 10,2 & 10,2 & 79,6 & 0,0 & 4,1 & 95,9 \\
\hline
\end{tabular}

NPC - Nenhum ou pouco conhecimento; RC - razoável conhecimento; BMC - bom ou muito conhecimento.

Nenhum dos estudantes demonstrou ter nível satisfatório de conhecimentos sobre Etnobotânica. Além disso, 24,5\%, $\mathrm{n}=12$ deles desconheciam sobre os cuidados que se deve ter ao utilizar plantas medicinais e somente $4,1 \%, \mathrm{n}=2$ tinham bom conhecimento sobre Fitoterapia. Porém, após o esclarecimento por meio da prática de ensino esses percentuais elevaram-se de forma expressiva para $51,0 \%, \mathrm{n}=25,81,6 \%, \mathrm{n}=40$ e $69,4 \%, \mathrm{n}=34$ respectivamente (Tabela 3 ).

Para Albuquerque e Hanazaki (2006) [21], a ausência de conhecimento em relação às características das plantas medicinais, seu uso com outros medicamentos, sem prescrição médica e, a ausência de instrução acerca dos efeitos terapêuticos e nocivos das plantas, e sua identificação taxonômica, são condições que preocupam, devido às pessoas utilizarem as plantas via automedicação.

Os estudantes apresentaram pouco conhecimento sobre a temática abordada de Etnobotânica. Esse conhecimento poderia subsidiar, e muito, o incremento de informações científicas sobre o uso das plantas. Para Brasileiro et al. (2008) [22], as plantas medicinais têm seu uso mais evidente na população mais idosa. Os alunos percebiam, mesmo antes da vivência didática, a Biologia como uma disciplina capaz de inserir os diversos aspectos (ecológicos, botânicos e taxonômicos) das plantas medicinais (Tabela 3). Esses saberes permitiriam um conhecimento mais abrangente e científico sobre as plantas e, consequentemente, a possível valorização delas no contexto Etnobotânico.

Em estudo semelhante realizado por Silva e Marisco (2013) [23] em uma escola pública no munícipio de Vitória da Conquista Bahia foi observado que $74,6 \%$ dos estudantes consideraram que o conhecimento em relação às plantas medicinais é importante para sua formação escolar. Dessa maneira, a escola pode ser mediadora significativa da postura crítica e reflexiva do aluno enquanto cidadão preocupado com a preservação das práticas e da ciência que a Etnobotânica envolve.

Para Venholi Junior e Vargas (2014) [24] ao inserir conhecimentos populares sobre plantas medicinais nas salas de aula, abrem-se oportunidades para o diálogo entre saberes trazidos pelo educando e o saber científico. Kovalski et al. (2011) [25] reiteram que os conhecimentos adquiridos pelos alunos são construídos no seu cotidiano e, por isso, precisam ser abordados no currículo escolar, porém tal conhecimento é deixado de lado. Para Silva e Nuñez (2007) [26] as concepções prévias são construídas pelo sujeito para compreender melhor o que acontece ao seu redor no dia a dia, e é através dessa concepção prévia que o professor vai elaborar as suas aulas e atender satisfatoriamente a necessidade dos estudantes. 
A prática didática com os alunos entrevistados proporcionou um aumento no percentual de estudantes que souberam conceituar corretamente o que é Etnobotânica e plantas medicinais (Tabela 4), correspondendo aos seguintes percentuais para as respostas certas: Etnobotânica antes, 18,4\% (n $=9) ; 75,5 \%(n=37)$ e plantas medicinais $28,6 \%(n=14)$; depois: $81,6 \%(n=40)$.

Tabela 4: Frequência percentual da classificação das respostas dos alunos entrevistados para os conceitos de Etnobotânica e Plantas medicinais

\begin{tabular}{ccccc}
\hline Etapa da pesquisa & Certo & Certo em partes & Errado & Não sei \\
\hline & \multicolumn{4}{c}{ plantas medicinais } \\
\hline Antes & 28,6 & 18,4 & 22,4 \\
Após & 81,6 & 75,5 & 6,1 & 0 \\
\hline \multicolumn{5}{c}{ Etnobotânica } \\
\hline Antes & 18,4 & 18,4 & 6,1 & 57,1 \\
Após & 75,5 & 2,0 & 4,1 & 18,4 \\
\hline
\end{tabular}

Os alunos conceituaram as plantas medicinais como sendo benéficas para saúde, onde 10,2\% (n = 21) deles afirmaram que "plantas medicinais servem para cura de doenças" e 5,4\% $(\mathrm{n}=11)$, destacaram plantas medicinais como "Plantas que servem como medicamento". Cruz et al. 2009 [18] também observaram que os alunos percebiam que as plantas medicinais são úteis para a cura de doenças. Na pesquisa realizada por Silva e Marisco (2013) [23], apenas 49,2\% dos estudantes usaram o conceito aproximado ao reportado pela Organização Mundial de Saúde (OMS), que refere as plantas medicinais como sendo útil para uso terapêutico.

Os estudantes demonstraram muito interesse ao participarem da pesquisa e interagiram durante toda a realização da prática didática. A abordagem de conteúdos que dialoga com o conhecimento pré-existente do educando faz com que conteúdos antes considerados irrelevantes passem a ter mais sentido para eles tornando as aulas mais dinâmicas e provocativas.

Dentre as partes mais utilizadas das plantas medicinais, a folha foi a mais citada $75,5 \%$ seguido de casca $63,3 \%$, raízes $57,1 \%$, sementes $28,6 \%$, fruto $12,2 \%$ e flor $2,0 \%$. Resultados similares, indicando a folha como a parte mais utilizada, também foram reportados por Soares et al. 2009 [27], com 73,0\% dos alunos citando as folhas; por Almeida et al. (2009) [28], com 72,0\% e por Lacerda et al. (2013) [29], com 87\% das citações.

Freitas et al. (2012) [30] relata que as folhas são a parte mais utilizada devido à maior disponibilidade de plantas herbáceas que apresentam folhas disponíveis todo ano. Pinto et al. (2000) [31] destaca que é relevante a identificação correta das partes a serem utilizadas, porque cada parte possui seu princípio ativo e pode também apresentar toxicidade.

Acerca dos efeitos medicinais 67,3\% dos alunos consideraram o efeito das plantas medicinais moderado, 30,6\% forte e 2,0\% muito forte; E após a realização da prática, as citações para moderado diminuíram para $51,0 \%$ e, as citações para forte $34,7 \%$ e muito forte $14,3 \%$ aumentaram consideravelmente. Isto é resultado da apresentação da temática ser abordada na prática de ensino, informando que a ação das plantas medicinais depende da maneira que se utiliza (Tabela 5). 
Tabela 5: Frequências absoluta ( $\mathrm{fa}$ ) e percentual ( $\mathrm{fr}$ ) das partes das plantas medicinais utilizadas por eles e quais o nível de efeito percebido

\begin{tabular}{lcc}
\hline Parte da planta & fa & fr \\
\hline Folhas & 37 & 75,5 \\
Cascas & 31 & 63,3 \\
Raizes & 28 & 57,1 \\
Semente & 14 & 28,6 \\
Fruto & 6 & 12,2 \\
Flor & 1 & 2,0 \\
\hline \multicolumn{3}{c}{ Nível do efeito } \\
\hline Moderado & 33 & 67,3 \\
Forte & 15 & 30,6 \\
Muito forte & 1 & 2,0 \\
\hline
\end{tabular}

Firmo et al. (2011) [32] explicam que são necessários estudos para esclarecer e validar as informações sobre os efeitos das plantas, tendo com o propósito diminuir efeitos adversos e intoxicação, para que tal uso seja eficiente e confiável.

Antes da prática didática, os estudantes $24,5 \%(\mathrm{n}=12)$ afirmaram que às plantas medicinais não causam nem um dano à saúde se utilizada de forma incorreta e 75,5\% $(\mathrm{n}=37)$ concordaram que quando utilizadas de forma errada, as plantas podem sim causar problemas de saúde. Após os esclarecimentos didáticos, por meio da palestra educativa, esses percentuais se alteraram para $8,2 \%$ $(n=4)$ e $91,8 \%(n=45)$, respectivamente.

Oliveira e Gonçalves (2006) [33] esclarecem que por acreditar que as plantas não causam problemas se forem utilizadas de forma incorreta, os alunos podem sofrer intoxicação pelo uso equivocado das plantas. Faz-se necessário um melhor entendimento sobre o uso dos recursos medicinais e os cuidados ao fazê-lo e o estudo das plantas medicinais e tóxicas para que juntamente com a percepção do estudante o conhecimento seja sistematizado de forma que o mesmo consiga levar para o seu convívio social.

Em relação à obtenção do conhecimento de plantas medicinais 51,0\% $(\mathrm{n}=25)$ dos alunos informaram ter herdado dos avós; 42,9\% $(n=21)$ dos pais; 4,1\% $(n=2)$, TV; $2,0 \%(n=1)$ outros. Rádio e livros não foram citados.

Cerca de 93,9\% ( $n=46)$ dos alunos informaram que a principal forma de obtenção desse conhecimento é através de familiares, mais precisamente avós, essa afirmação podemos observar em Ferreira et al. (2015) [34], quando dizem que:

Dos pesquisados, cerca de $80 \%$ afirmaram ter adquirido os conhecimentos sobre plantas com potenciais medicinais através da cultura transmitida pelos avós, seguido pelos pais [...]. Os cuidados quanto à utilização de plantas medicinais também foram enfatizados pelos entrevistados. (p. 67, 2015)[34].

O estudo Etnobotânico no meio escolar evidencia uma nova forma de abordagem de conteúdos externos ao currículo levando em consideração o saber acumulado pelos alunos em seu meio cultural e social. Os educadores ao utilizarem-se dessa ferramenta estão buscando uma estratégia que auxilia na aprendizagem do educando, de maneira que possam tornar-se indivíduo capaz de refletir e compreender o que o cerca de forma crítica. 


\section{CONCLUSÃO}

Antes da prática de ensino os estudantes não apresentaram conhecimento satisfatório sobre plantas medicinais e o seu uso. A prática didática sensibilizou os alunos para mediar o conhecimento, aonde a análise da reaplicação do questionário reporta diferenças significativas para melhor, quanto ao saber dos alunos sobre a Etnobotânica e plantas medicinais.

Dessa maneira, observa-se que a inserção do ensino sobre plantas medicinais no contexto escolar, propicia a construção do saber e, consequentemente, melhor valorização e uso dessas plantas presentes na comunidade em que os alunos vivem.

\section{AGRADECIMENTOS}

A direção e aos alunos da Escola Estadual de Ensino Fundamental e Médio Francisco Romano da Silveira e a todos que contribuíram direta ou indiretamente com essa pesquisa.

\section{REFERÊNCIAS BIBLIOGRÁFICAS}

1. Boscolo OH. Para comer, para beber ou para remédio? Categorias de uso múltiplo em Etnobotânica. Centro Universitario de Volta Redonda- Volta Redonda - Rio de Janeiro. 2013;1(1):61-67.

2. Quinteiro MMC, Tamashiro AMG, Moem GM. Etnobotânica à comunidade no paradigma da complexidade ambiental e educação ambiental. Revista Brasileira de Educação Ambiental. 2013;8(1):91-99.

3. Gottlieb OR, Borin MRMB, Pagotto CLAC. Biodiversidade: o enfoque interdisciplinar brasileiro. 1988;3(2):97-102.

4. Bessa NGF, Borges JCM, Beserra FP, Carvalho RHA. Prospecção fitoquímica preliminar nativas do cerrado de uso popular medicinal pela comunidade rural do assentamento vale de plantas verde - Tocantins. Revista Brasileira de Plantas Medicinais. 2013;15(4):692-707.

5. Santana RS, Bianchini-Pontuschkal BR, Hurtado BF, Olivera AC. Uso medicinal do óleo de copaíba (Copaifera sp.) por pessoas da melhor idade no município de Presidente Médici, Rondônia, Brasil. Revista Acta Agronómica. 2014;63(4):361-366.

6. Silva NLA, Miranda FAA, Conceição GM. Triagem fitoquímica de Plantas do Cerrado da Área de Proteção Ambiental Municipal do Inhamum, Caxias, Maranhão. Revista Scientia Plena. 2010;6(2):1-17.

7. Roque AA, Rocha RM, Loiola MIB. Uso e diversidade de plantas medicinais da Caatinga na comunidade rural de Laginhas, município de Caicó, Rio Grande do Norte (nordeste do Brasil). Revista. Brasileira de Plantas Medicinais. 2010;12(1):31-42, doi: 10.1590/S1516-0572 2010000100006.

8. Brasil. Ministério da Saúde aprova a Política Nacional de Plantas Medicinais e Fitoterápicos e dá outras providências. Secretaria de Ciência, Tecnologia e Insumos Estratégicos, Departamento de Assistência Farmacêutica 2006; 63 p. Disponível em: http: <//portal. saude. gov. br/portal/arquivos/pdf/Decreto_Fito .pdf>. Acessado em 20 de fevereiro de 2016.

9. Dávila ES, Alves CC, Lima BM, Folmer V. Ideias prévias sobre plantas medicinais e tóxicas de estudantes do ensino fundamental da região da Fronteira Oeste do Rio Grande do Sul. Revista de Linguagens, Artes e Estudos em Cultura. 2016;2(1):358-368.

10. Costa RGA. Os saberes populares da etnociência no ensino das ciências naturais: uma proposta didática para aprendizagem significativa. Revista Didática Sistêmica. 2008;8.

11. Paiva AS, Almeida RO, Martins KV. Ciência e outras culturas: proposições para o ensino de ciências e biologia. $4^{\circ}$ Congresso Ibero-Americano em Investigação Qualitativa - $4^{\circ}$ CIAIQ / $6^{\circ}$ Simpósio Internacionalde Educação e Comunicação - $6^{\circ}$ SIMEDUC. 2015; 2: 390-393. Disponível em: $<$ http://proceedings. ciaiq. org/ index. php/ ciaiq 2015/ article/viewFile/326/321>. Acessado em 16 de Abril de 2016.

12. Amadeu SO, Maciel ML. A dificuldade dos professores de educação básica em implantar o ensino prático de Botânica. Revista de Produção Discente em Educação Matemática. 2014;3(2):225-235.

13. IBGE - Instituto Brasileiro de Geografia Estatística. Disponível em:< http:// www. cidades. ibge. gov. $\mathrm{br} / \mathrm{xtras} /$ home .php? codnum=250> . Acessado em 28 de Abril de 2016.

14. Rocha JSM. Manual de Projetos Ambientais. Santa Maria: UFSM, 1997; 446. 
15. Likert R. Technique for the measurement of atitudes. Archives of psychology. 1932;22(140):1-55. Disponível em: http://www.voteview.com/pdf/Likert_1932.pdf. Acessado em: 19 de Maio de 2017.

16. Albuquerque UP. Introdução a etnobotânica. $2^{\mathrm{a}}$ Edição, 2005. 93 p.

17. Brasil. Ministério da Saúde Resolução ANVISA - RDC nº 10, de 9 de março de 2010. Disponível em: portal.anvisa.gov.br/../RDC_10_2010.../6d4feca0-9b45-48f8-b44e-231fa048e4a6? Acessado em 28 de Abril de 2016.

18. Cruz LP, Furlan MR, Joaquim WMO. Estudo de plantas medicinais no ensino fundamental: uma possibilidade para o ensino da botânica. VII Encontro Nacional de Pesquisa em Educação em Ciências ENPEC. 2009. Disponível em: 〈http://posgrad. fae. ufmg.br/posgrad/ viienpec/ pdfs/270.pdf〉. Acessado em 28 de Abril de 2016. ISSN 217669-40.

19. Veiga Junior VF, Pinto AC, Maciel MAM. Plantas medicinais: cura segura? Química Nova. 2005;28(3):519-528, doi:10.5935/0100-4042.20160050.

20. Fundação Oswaldo Cruz. Sistema Nacional de Informações Tóxico - farmacológicas (FIOCRUZ/SINITOX). Casos Registrados de Intoxicação Humana por Agente Tóxico e Zona de Ocorrência. Brasil, 2012. Disponível em: <http://www. fiocruz.br/sinitox/media /Tabela\%203_2012. Pdf.> Acessado em 18 de Abril de 2016.

21. Albuquerque UP, Hanazaki N. As pesquisas etnodirigidas na descoberta de novos fármacos de interesse médico e farmacêutico: fragilidades e perspectivas. Revista Brasileira de Farmacognosia. 2006;16(Supl):678-689.

22. Brasileiro BG, Pizziolo VR, Matos DS, Germano AM, Jamal CM. Plantas medicinais utilizadas pela população atendida no "Programa de Saúde da Família", Governador Valadares, MG, Brasil. Revista Brasileira de Ciências Farmacêutica. 2008;44(4): 629-636.

23. Silva TSS, Marisco G. Conhecimento etnobotânico dos alunos de uma escola pública no município de Vitória da Conquista/Ba sobre plantas medicinais. Revista de Biologia e Farmácia. 2013;9(2):62-73.

24. Vinholi Júnior AJ, Vargas IA. Saberes tradicionais sobre plantas medicinais: interfaces com o ensino de botânica. Imagens da Educação. 2014;4(3):37-48.

25. Kovalski ML, Obara AT, Figueiredo MC. Diálogo dos saberes: o conhecimento científico e popular das plantas medicinais na escola. VIII Encontro Nacional de Pesquisa em Educação em Ciências - ENPECABRAPEC. 2011. Disponível em: <http://www.nutes.ufrj.br/abrapec/viiienpec/ lista_area_12. htm.>. Acessado em 29 de Abril de 2016.

26. Silva MGL, Núñez IB. Concepções alternativas dos estudantes. Programa Universidade a Distância UNIDIS Graduação. 2007. 15 p.

27. Soares MAA, Braga JRP, Mourão AEB, Parente KMS. Levantamento etnobotânico das plantas medicinais utilizadas pela população do município de Guirnhém-Paraiba. Revista Homem, Espaço e Tempo. 2009;3647.

28. Almeida NFL, Silva SRS, Souza JM, Queiroz APN, Miranda GS, Oliveira HB. Levantamento etnobotânico de plantas medicinais na cidade de Viçosa - MG. Revista Brasileira de Farmácia. 2009;90(4):316-320.

29. Lacerda JRC, Sousa JS, Sousa LCFS, Borges MGB, Ferreira RTFV, Salgado AB, Silva MJS. Conhecimento popular sobre plantas medicinais e sua aplicabilidade em três segmentos da sociedade no município de Pombal-PB. Agropecuária Científica no Semiárido. 2013;9(1):14-23.

30. Freitas AVL, Coelho MFB, Maia SSS, Azevedo RAB. Plantas medicinais: um estudo etnobotânico nos quintais do Sítio Cruz, São Miguel, Rio Grande do Norte, Brasil. Revista Brasileira de Biociências. 2012;10(1):48-59.

31. Pinto JEBP, Santiago EJA, Lameira OA. Plantas Medicinais. Lavras: PROEX/ UFLA, 2000. 74p. (Boletim Extensão, 70).

32. Firmo WCA, Menezes VJ M, Passos CEC. Contexto histórico, uso popular e concepção científica sobre plantas medicinais. Caderno de Pesquisa. 2011; 18(especial):90-95.

33. Oliveira FQ, Gonçalves LA. Conhecimento sobre plantas medicinais e fitoterápicos e potencial de toxidade por usuários de Belo Horizonte, Minas Gerais. Revista Eletrônica de Farmácia. 2006;3(2):3641.

34. Ferreira ALS, Batista CAS, Pasa MC. Levantamento etnobotânico nas diferentes realidades de ensino Revista Biodiversidade. 2015;14(3):60-73. 